\title{
How Multi-services Gateways Enable loT for Utilities
}

\author{
Regis Hourdouillie ${ }^{1}$ and Don Pollock ${ }^{2}$ \\ 1. Customer Group Industry and Society, Ericsson France, 91348 MASSY Cedex, France \\ 2. Customer Group Industry \& Society, Ericsson Ltd., Guildford, GU2 8SG, UK
}

Received: July 22, 2016 / Accepted: August 09, 2016 / Published: November 30, 2016.

\begin{abstract}
Modern internet of things technologies bear the promise of cheap, ubiquitous and standard solutions for the smart grid. The end-to-end architecture relies upon transverse service enablement and device connectivity platforms, LTE communications solutions and communication gateways bridge the gap between 3GPP standards and legacy communications solutions. This paper will have a specific focus on the multi-services, multi-protocols gateways that allow connection to legacy assets, and how they are a critical component for the digitalization of power grids and cities. We will illustrate how this gateway — called the Smart Grid Node- has enabled Duke Energy (USA) to initiate its grid modernization project in Cincinnati, Ohio. We will also show that the smart grid node is much more than a communication gateway as it can host very diverse decentralized applications and therefore be a key component to enable multi-services smart city nodes in the near future.
\end{abstract}

Key words: Smart grid, utility communications, telecommunications, IoT, gateway, grid edge, operational efficiency.

\section{Introduction}

Distribution grid operators are working in a dynamic context: Customers are demanding greater understanding and control over their energy usage, Governments are pushing for cleaner sources of energy generation. Reliability and safety are, as ever, paramount. Integration of distributed generation increases the need for grid visibility and rapid response to local grid disturbances. Load patterns are shifting and (in some countries) there is aging infrastructure. All the while, technology continues to advance offering new solutions for grid management and there is increased capability and challenges from the proliferation of intelligent devices through; smart metering, demand response, electric vehicles, grid scale storage, distribution automation, distributed generation and intelligent streetlight control. Collectively these (and others) are all covered by the umbrella: Utility IoT (internet of things).

A comprehensive and forward-looking IoT technology strategy must be developed prior to

Corresponding author: Don Pollock, B.Sc. (Hons), global offering lead utility communications, smart grid. embarking on deployment to fully leverage new investments, and to embrace the value of legacy systems. This strategy should promote the flexibility of existing operating resources to incorporate future applications and end-point types as they emerge, and as the smart grid or city develops.

Distribution grid operators need cost effective control and communication architectures that take account of the dynamic context. Utility IoT may consist of thousands of communicating devices. Enabling numerous new devices also creates an exponential increase in the volume of new data generated from those devices. Certainly, These data will help an utility to understand its distribution grid operations at a granular level, which will enable more targeted operational decisions and ultimately more efficient operation of the distribution grid. As a consequence, the process of managing this data flow, and analysis, will be integral to how Utility IoT benefits are realized.

\section{Utility Challenges}

\subsection{Knock Down the Silos}

The prevailing norm in utilities was and in some 
cases still is the deployment of disparate systems and devices that are independent of each other, each managed separately and often using an array of communications technologies, sometimes with proprietary protocols.

The data generated within these disparate systems are typically collected, transmitted to and analyzed at a central location with control decisions coming from the same central location. Integration of data from different systems can be costly and time consuming.

\subsection{Balancing Lifecycles and Evolution of Utility} Assets and Telecommunications

Typically, utility assets have long lifetimes but telecommunications develop at a faster pace. Embedding communications in assets makes it difficult to take advantage of new telecommunications technology as it becomes available. It is essential for distribution grid operators to optimize both capital and operating expenditure when implementing regulated or necessary modernization initiatives. To do so means: Maintaining the value of and avoid stranding legacy assets; Providing for differing latencies for communications and control for different applications on the grid; Avoiding duplication of assets; their function and management; Avoiding application specific single vendor lock-in.

It is also essential to prepare for future communications requirements and applications. An incremental and disconnected approach to IoT strategy proves too costly and ineffective. An integrated implementation strategy that adheres to a common infrastructure model will yield significant advantages.
There is a new paradigm for grid management that can equally be applied to smart city development.

\section{Multi-services Gateways}

Learning from best practice suggests a better approach to IoT implementation, one that emphasizes system-wide common architectures. An approach that is capable of pushing data collection, analysis, and application out to the edge of the grid while leveraging multiple communications technologies. This approach can maximize value by; reducing the cost of implementation, communications and operations, delivering network visibility and control, providing for new applications and technology through a flexible foundation, incorporating and extending the value of legacy assets.

Effective communications are essential for realizing the operational and financial benefits associated with the inexorable march towards Utility IoT. It is vital to have communications that offer the reliability, bandwidth, latency and cost performance suitable for each application.

Multi-services gateways provide a platform approach to Utility IoT, comprising both hardware and software, that offers choice of communications interfaces to enable multiple distribution grid applications, in parallel, on a single common infrastructure model (Fig. 1). They (and appropriate downstream devices) are managed by a dedicated element management system. We call these devices the Smart Grid Node, and they provide the physical control, logical and telecommunications hub (or access points) for the distribution grid.

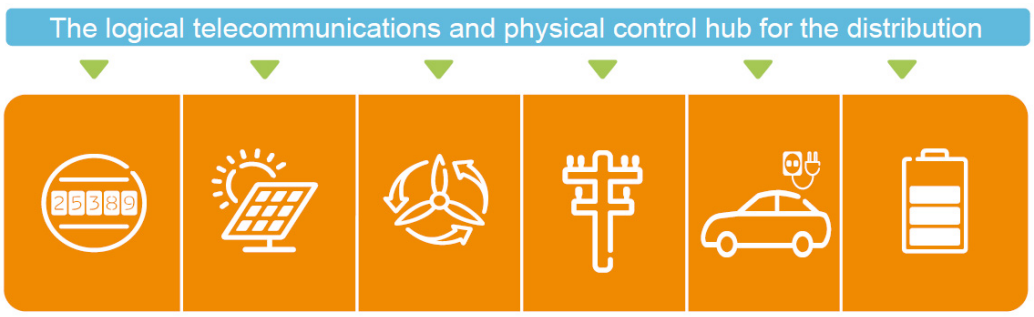

Common communications architecture

Fig. 1 Common communications infrastructure. 


\subsection{Standards Really Matter}

Today's lower cost proprietary solutions may very well be tomorrow's restrictive and inefficient problems. That is why adopting a standards approach to smart grid communications makes sense. In addition, commonly supported standards will promote interoperability and afford choice of vendors helping to push down cost. Using open source operating system allows maximum flexibility for application development by multiple parties.

These standards-based nodes enable secure two-way, near real-time communication, providing data entry \& control points anywhere on a distribution system. They can host and/or integrate developed applications, and are remotely upgradable. The 3GPP standards-based versions connect to whichever $(4 \mathrm{G}, 3 \mathrm{G}$ or $2 \mathrm{G})$ cellular network is available and GPS provides precise location of grid assets.

Interoperability and legacy asset inclusion are facilitated by protocol support and conversion (e.g. IEC 61850, IEC 60870-5-101/4, DNP3, ModBus and MQTT). Interoperability between systems provides the means to leverage data from multiple sources to better effect both centrally and at the edge of the grid.

\subsection{Connectivity Choice}

For connectivity, the Smart Grid Nodes can support a variety of standard wired and wireless interfaces, such as: Serial (EIA 232, EIA 485), Ethernet, ISM band Radio Frequency, IEEE 802.11 Wi-Fi, Cellular (2G, $3 \mathrm{G}$ and $4 \mathrm{G}$ networks) utilizing multiple wireless carriers, and OFDM PLC (power line communications) technology (PRIME and G3-PLC Standards). The modular nature of the nodes facilitates the addition of other standards-based communications technologies as they evolve.

\subsection{Distributed Intelligence-A Positive Impact on Data Costs}

Most utility grid applications focus on collection and transportation of data from field devices back to a central data warehouse, where analysis is performed by the application's head-end management system. Recent industry discussions of analytics focus primarily on extracting data from the data warehouse for incremental analysis beyond what each individual application may be capable of. There is value in centralizing data storage and analytics. There is also value in performing analysis of data at the edge, where the data originate. This has value to reduce communication costs associated with transporting data back to a central data warehouse and to optimize data warehouse storage costs. This distributed intelligence-An architecture with intelligence at the grid edge offers the potential for new solutions and benefits.

\subsection{Dedicated Element Management}

Communications network complexity is reduced by having a single, but extensible, element management software platform (Fig. 2) for multiple applications that manages the communications network of these multi-purpose gateways between Utility IoT devices and the existing back-office systems. Again a standards-based approach is adopted through the use of SNMP (simple network management protocol). It provides full FCAPS capability. FCAPS is the ISO Telecommunications Management Network model and framework for network management. FCAPS is an acronym for fault, configuration, administration, performance, security, the management categories into which the ISO model defines network management tasks.

As with many IoT solutions there is choice to where the management solution is hosted: internal servers or cloud-based.

\subsubsection{Reducing Complexity}

The element management system is the "glue" that binds the diverse Utility IoT application areas and makes possible the reduction in network complexity. It can monitor and manage tasks, supports auto-discovery 

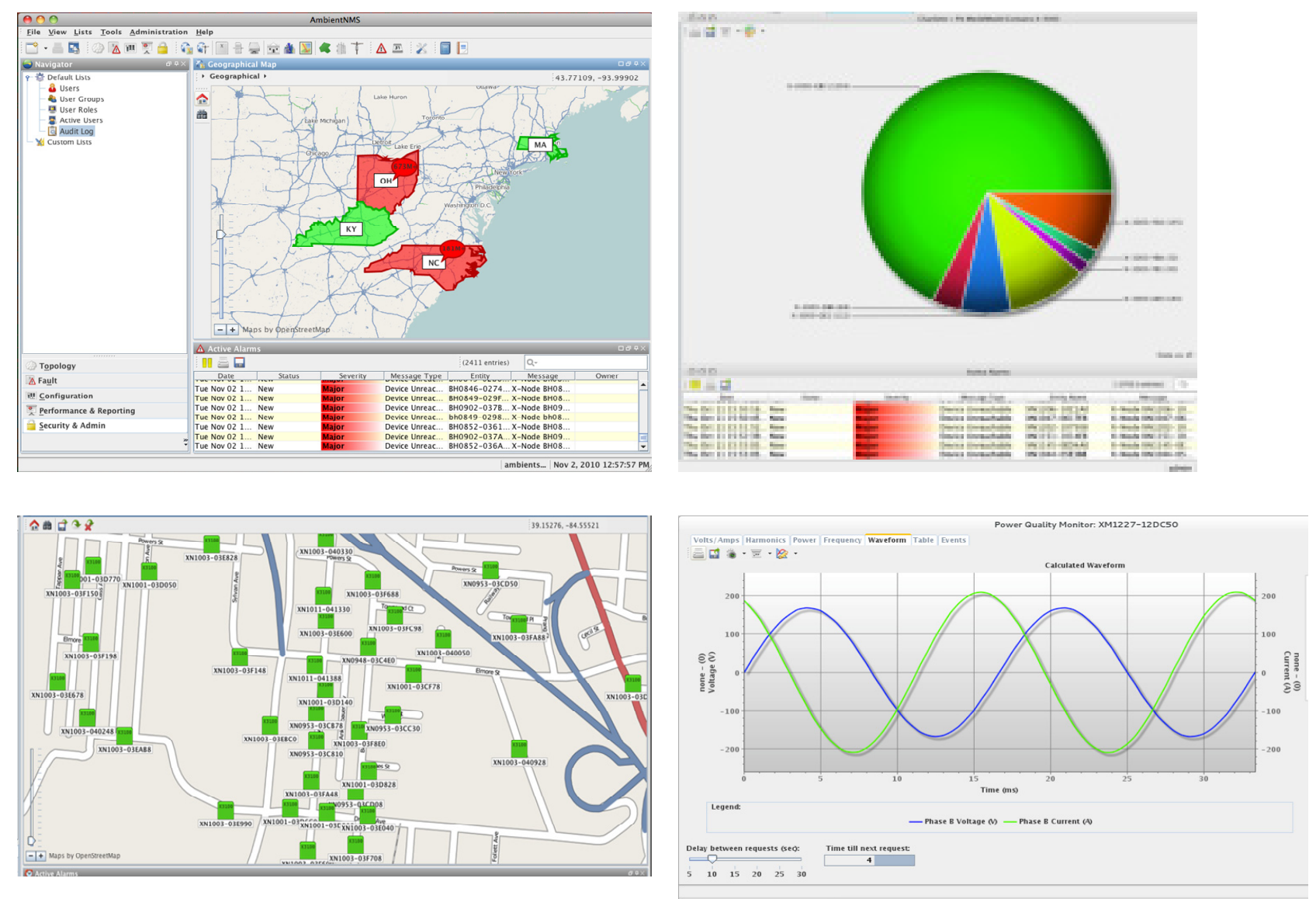

Fig. 2 Element management software platform for multiple applications.

of network devices without user input/intervention, and performs status checks, queries devices and stores and relays information and instructions between the management system and the nodes. It can manage distribution of software upgrades to the nodes and other downstream devices manufactured by others that conform to existing standards. Additionally, it provides secure tools for data reporting including alerts, alarms (configurable based on user-defined thresholds), event logging, data collection, and custom reporting, as well as interpreting, arranging, and sharing data. The management system allows the utility to define and limit user access as needed, and users can customize their experience to their roles, tasks, and priorities. It provides high visibility of grid assets through customizable lists and maps; geographic maps with enhanced views of electrical substation and circuit topology. Vitally, in the ever-burgeoning world of
Utility IoT it supports multiple levels of scaling and can be integrated with other utility operational systems through standard protocol interfaces.

\section{An Integrated Approach to Smart Grid- Duke Energy}

Duke Energy, a US investor owned utility, is one of the largest electric power companies in the US. Starting in 2009, it conducted a comprehensive grid modernization program, funded in part by the SGIG (smart grid investment grant) under the American Recovery and Reinvestment Act of 2009.

The Smart Grid Node played a role in this program enabling multiple services (predominantly in Cincinnati, Ohio); connectivity to grid monitoring (MV line sensors) and control assets (capacitor banks, voltage regulators), providing a common aggregation point for residential electric and gas smart metering (Fig. 3), 


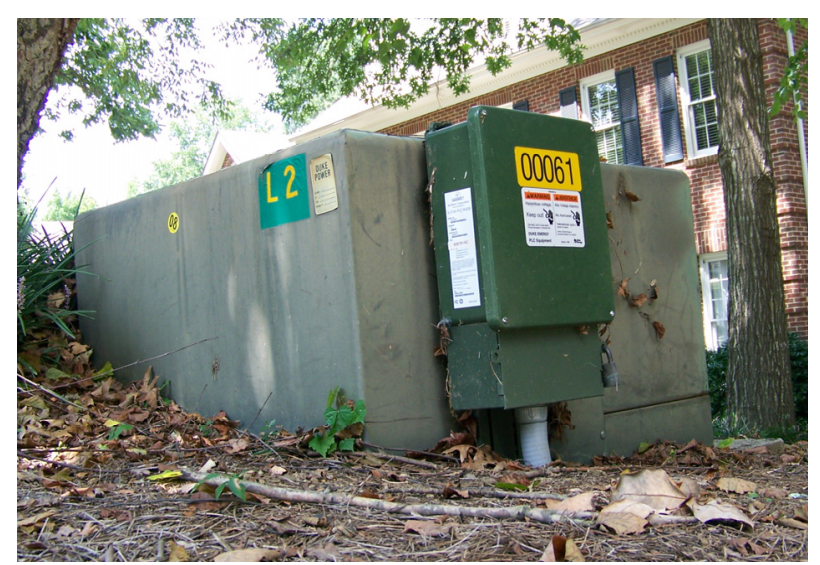

Fig. 3 Common aggregation point for residential electric and gas smart metering.

enabling improvements in outage detection and restoration, integrated streetlight control, weather sensors and serial devices for distribution automation.

The latest development work undertaken by Duke and others continues to move away from the traditional centralized proprietary systems and towards support of distributed intelligence, interoperability and utility IoT. These concepts enabled through what the industry calls OpenFMB (open field message bus). A concept was showcased in a collaborative demonstration at the DistribuTECH 2016 meeting in Orlando, Florida, U.S.

\section{Conclusions}

The key business benefit offered by multi-services, multi-protocol IoT gateways for utilities is the ability to drive down the cost of grid modernization, which in turn accelerates return on investment.

\subsection{Multiple-purpose Single Infrastructure}

Using the same infrastructure for multiple applications on the distribution grid reduces the cost of
Utility IoT implementation in a number of ways: A shared means of backhaul and communications management eliminates single use networks, reducing the number of devices in the field, cost and complexity. A whole network view leads to faster problem resolution and allows a single schema for ensuring security of data. A single point of responsibility for communications across multiple applications reduces field install costs and can accelerate IoT deployment. A multi-function, communications platform that facilitates the integration of legacy and new devices and allows for a phased approach to building out new applications. In addition, it provides the foundation for future applications.

Doubtless there will be advances in technology, and having a communications platform that is sufficiently flexible to incorporate existing and future applications will provide financial benefits. A system wide communications infrastructure not dedicated to individual end-point devices will allow a "refresh" instead of full replacement making the most of grid assets.

Applying this approach to Smart Cities will doubtless realize similar benefits.

\section{References}

[1] Pollock, D. 2014. "Boosting Power Grid Resilience-Communications for Real-Time Network Visibility." Electricity Today 27 (3): 8-12.

[2] U.S. Department of Energy. 2014. "Integrated Smart Grid Provides Wide Range of Benefits in Ohio and Carolinas." Smart Grid Investment Grant Program.

[3] Laval, S., and Malcolm, W. P. 2016. "Duke Develops True Interoperability.” T\&D World Magazine 68 (3): $70-5$. 